$1-28-2013$

\title{
The Very Short Period M Dwarf Binary SDSS J001641-000925
}

James R. A. Davenport

Western Washington University, james.davenport@wwu.edu

Andrew C. Becker

Andrew A. West

John J. Bochanski

Suzanne L. Hawley

See next page for additional authors

Follow this and additional works at: https://cedar.wwu.edu/physicsastronomy_facpubs

Part of the Stars, Interstellar Medium and the Galaxy Commons

\section{Recommended Citation}

Davenport, James R. A.; Becker, Andrew C.; West, Andrew A.; Bochanski, John J.; Hawley, Suzanne L.; Holtzman, Jon; Gunning, Heather C.; Hilton, Eric J.; Munshi, Ferah A.; and Albright, Meagan, "The Very Short Period M Dwarf Binary SDSS J001641-000925" (2013). Physics \& Astronomy. 19.

https://cedar.wwu.edu/physicsastronomy_facpubs/19 


\section{Authors}

James R. A. Davenport, Andrew C. Becker, Andrew A. West, John J. Bochanski, Suzanne L. Hawley, Jon Holtzman, Heather C. Gunning, Eric J. Hilton, Ferah A. Munshi, and Meagan Albright 


\title{
THE VERY SHORT PERIOD M DWARF BINARY SDSS J001641-000925*
}

\author{
James R. A. Davenport ${ }^{1}$, Andrew C. Becker ${ }^{1}$, Andrew A. West ${ }^{2}$, John J. Bochanski ${ }^{3}$, Suzanne L. Hawley ${ }^{1}$, \\ Jon Holtzman ${ }^{4}$, Heather C. Gunning ${ }^{1}$, Eric J. Hilton ${ }^{5}$, Ferah A. Munshi ${ }^{1}$, and Meagan Albright ${ }^{1}$ \\ ${ }^{1}$ Department of Astronomy, University of Washington, Box 351580, Seattle, WA 98195, USA; jrad@ astro.washington.edu \\ 2 Astronomy Department, Boston University, 725 Commonwealth Avenue, Boston, MA 02215, USA \\ ${ }^{3}$ Astronomy and Astrophysics Department, Pennsylvania State University, 525 Davey Laboratory, University Park, PA 16802, USA \\ ${ }^{4}$ Department of Astronomy, New Mexico State University, Box 30001, Las Cruces, NM 88003, USA \\ ${ }^{5}$ Department of Geology and Geophysics and Institute for Astronomy, University of Hawaii at Manoa, Honolulu, HI 96822, USA \\ Received 2012 June 22; accepted 2012 December 21; published 2013 January 28
}

\begin{abstract}
We present follow-up observations and analysis of the recently discovered short period low-mass eclipsing binary, SDSS J001641-000925. With an orbital period of 0.19856 days, this system has one of the shortest known periods for an M dwarf binary system. Medium-resolution spectroscopy and multi-band photometry for the system are presented. Markov Chain Monte Carlo modeling of the light curves and radial velocities yields estimated masses for the stars of $M_{1}=0.54 \pm 0.07 M_{\odot}$ and $M_{2}=0.34 \pm 0.04 M_{\odot}$, and radii of $R_{1}=0.68 \pm 0.03 R_{\odot}$ and $R_{2}=0.58 \pm 0.03 R_{\odot}$, respectively. This solution places both components above the critical Roche overfill limit, providing strong evidence that SDSS J001641-000925 is the first verified M-dwarf contact binary system. Within the follow-up spectroscopy we find signatures of non-solid body rotation velocities, which we interpret as evidence for mass transfer or loss within the system. In addition, our photometry samples the system over nine years, and we find strong evidence for period decay at the rate of $\dot{P} \sim 8 \mathrm{~s} \mathrm{yr}^{-1}$. Both of these signatures raise the intriguing possibility that the system is in over-contact, and actively losing angular momentum, likely through mass loss. This places SDSS J001641-000925 as not just the first M-dwarf over-contact binary, but one of the few systems of any spectral type known to be actively undergoing coalescence. Further study of SDSS J001641-000925 is ongoing to verify the nature of the system, which may prove to be a unique astrophysical laboratory.
\end{abstract}

Key words: binaries: eclipsing - binaries: spectroscopic - stars: low-mass

Online-only material: color figures

\section{INTRODUCTION}

M dwarfs, with masses between $\sim 0.08$ and $0.6 M_{\odot}$, are the most numerous and faintest main-sequence stars in the Galaxy. The high number density and very long main-sequence lifetimes of $\mathrm{M}$ dwarfs allow them to be used as detailed tracers of the nearby galactic stellar population (Bochanski et al. 2007a, 2010). These stars are also well known for their high magnetic activity levels, characterized by frequent violent flaring outbursts (e.g., Kowalski et al. 2009; Davenport et al. 2012), and $\mathrm{H} \alpha$ emission (West et al. 2008, 2011; Hilton et al. 2010).

Eclipsing binary systems provide us with the valuable ability to directly measure the masses and radii of the two component stars. As discussed in Becker et al. (2011), the sample of known binary systems composed of two M dwarfs is very small. Their intrinsically low luminosity and small radii conspire to make M dwarf binaries difficult to detect in variability surveys. The fraction of $\mathrm{M}$ dwarfs in binary systems is also much lower than for higher mass stars. Lada (2006) has estimated that as few as $\sim 10 \%$ of $\mathrm{M}$ dwarfs in the Galactic field may be in binary (or higher number) configurations.

\footnotetext{
* Based on observations obtained with the Apache Point Observatory $3.5 \mathrm{~m}$ telescope, which is owned and operated by the Astrophysical Research Consortium. This paper includes data gathered with the $6.5 \mathrm{~m}$ Magellan Telescopes located at Las Campanas Observatory, Chile. Support for the design and construction of the Magellan Echellette Spectrograph was received from the Observatories of the Carnegie Institution of Washington, the School of Science of the Massachusetts Institute of Technology, and the National Science Foundation in the form of a collaborative Major Research Instrument grant to Carnegie and MIT (AST0215989).
}

The M dwarf binary and single-star systems that are well studied indicate that stellar structure models do not accurately predict the observed radii (Ribas 2006; Morales et al. 2010). Radii are often observed to be $\sim 10 \%-20 \%$ larger than predicted for a given mass. This may be due to limitations of the stellar models used, or to a fundamental difference in the interior structure of M dwarfs. Magnetic activity is frequently identified as a potential culprit, causing the radii to be enlarged due to the inhibition of convection or decreased heat flux from magnetic spots (e.g., Chabrier et al. 2007; Bochanski et al. 2011). Long period $\mathrm{M}$ dwarf binaries ( $P \gtrsim 10$ days) are ideal systems for radii determinations, as they are not influenced by spin-orbit coupling or tidal distortion (Mazeh 2008). The above discrepancies will only be resolved by the addition of many accurately measured radii to the known sample over a wide range in mass and period.

There also appears to be an absolute lower limit on the orbital period for contact binary star systems, though no consensus about its origin has been reached. This short period limit at $\sim 0.22$ days may be due to both components being fully convective (Rucinski 1992). At shorter periods, these stars cannot adhere to their mass-radius relationship while simultaneously staying within the Roche equipotential geometry. However, as Rucinski (1992) points out, this does not appear to be the primary effect at work in creating the sharp 0.22 day period limit. Alternatively, Jiang et al. (2012) have shown that unstable mass transfer for low-mass contact binaries may result in rapid coalescence. The observed period limit would thus be a result of the very short lifetimes for such systems. Models of angular momentum loss in binary systems, however, indicate that even given favorable mass 
ratios and initial orbital periods, 6-13 Gyr timescales are typically required for low-mass binaries to obtain periods as short as 0.22 days (Stepien 2006b). The observed period limit would then be a consequence of the finite age of the binary population.

Until recently, the shortest known $M$ dwarf binary system period in the literature was BW3 V38 (Maceroni \& Rucinski 1997; Maceroni \& Montalbán 2004), a detached binary composed of two main-sequence M3 dwarfs with an orbital period of 0.1984 days. Rucinski \& Pribulla (2008) found a similarly short period system, GSC $01387-00475$, with a period of 0.2178 days, but composed of higher mass K3 and K5 stars and in a contact configuration.

Dimitrov \& Kjurkchieva (2010) reported the shortest period M dwarf binary yet characterized, GSC 2314-0530 with a 0.192636 day period, and stellar masses of 0.51 and $0.26 M_{\odot}$. This system has an orbital period only $\sim 8$ minutes shorter than that of our target, SDSS J001641-000925. Nefs et al. (2012) have recently discovered four binary systems with $M$ dwarf colors that have periods significantly below the $\sim 0.22$ day period limit, providing a major challenge to our understanding of the formation and lifetimes of such systems. The rarity of short period $\mathrm{M}$ dwarf binaries, and the lack of known contact $\mathrm{M}$ dwarf binary systems, indicates that they are difficult to produce, are so unstable that they are rapidly destroyed, or are still very difficult to detect.

A recent study of photometric variability by Becker et al. (2011, hereafter B11) using the Sloan Digital Sky Survey (SDSS; York et al. 2000) revealed several new candidate $\mathrm{M}$ dwarf-M dwarf eclipsing binary systems. B11 analyzed $\sim 4.8$ million light curves and found 207 periodically variable objects, and a sharp drop in the number of systems with periods less than 0.22 days. Of these, two candidate eclipsing binary systems with sinusoidal light curves and $\mathrm{M}$ dwarf ugriz colors were found to have very short periods: SDSS J200011.19+003806.5 at 0.14552 days and SDSS J001641.03-000925.2 at 0.198561 days. Both of these objects warranted spectroscopic and photometric follow-up to determine their fundamental parameters, and to confirm their status as short period eclipsing binaries.

In this paper we describe our detailed photometric and spectroscopic investigation of the brighter of these two objects, the 0.198561 day periodic variable SDSS J001641-000925. In Section 2 we describe the photometric and spectroscopic data used in this study. We model basic parameters in Section 3, and analyze the peculiarities of the binary system in Section 4 . We discuss the context of this over-contact binary in Section 5.

\section{OBSERVATIONS}

SDSS J001641-000925 was discovered as a periodic variable object by B11 using time-domain data from the SDSS "Stripe 82" region (Ivezić et al. 2007; Sesar et al. 2007). This unresolved binary system has an SDSS ObjID $=587731186205720773$ and SpecObjID $=$ 314914764310446080 . A period of 0.198561 days was determined from 61 epochs of griz photometry, spanning more than eight years of observations, using the SuperSmoother algorithm (Friedman 1984; Reimann 1994). This algorithm does not assume a light curve shape, and instead works to find the period that minimizes the phase folded light curve dispersion. SDSS J001641-000925 has a median $r$-band magnitude of 16.39. A photometric spectral type of M1 was found using the median riz bands and the covariance matrix technique from Kowalski et al. (2009), while a type of M0 was assigned using

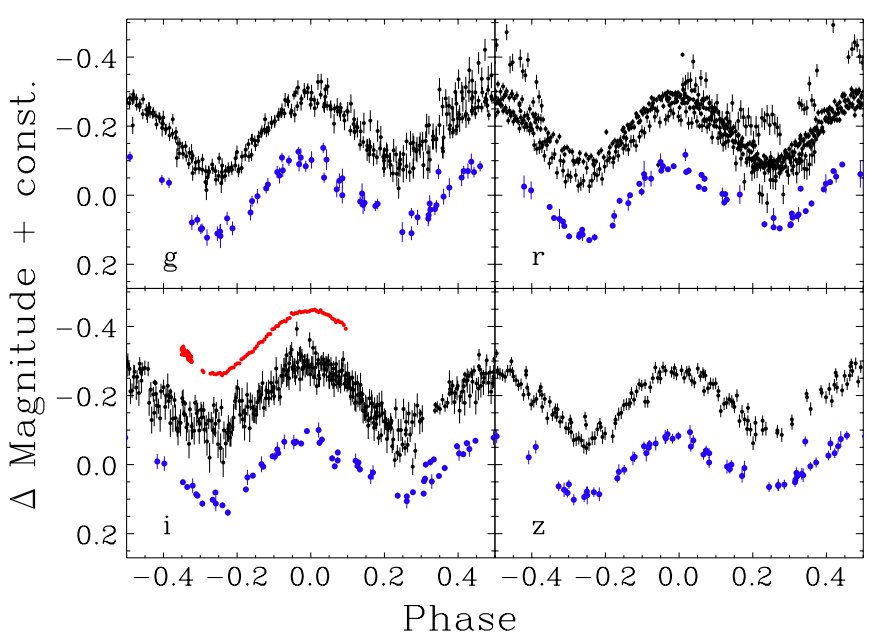

Figure 1. Phased light curves in the five SDSS filter bandpasses for SDSS J001641-000925. Photometric errors are shown for all data. Blue points are from the Stripe 82 catalog, black are follow-up data from the NMSU $1 \mathrm{~m}$ in griz bands. Red data in the $i$ band were obtained with the Agile camera at the APO $3.5 \mathrm{~m}$.

(A color version of this figure is available in the online journal.)

the "Hammer" spectral-typing analysis package (Covey et al. 2007), which compares spectral data to a set of standard templates. The SDSS spectrum did not show any clear signatures of binarity, such as spectral line separation or large deviation from the M0 spectral template (Bochanski et al. 2007b). In the following section, we describe follow-up data obtained to characterize this short period object.

\subsection{Photometry}

Figure 1 contains all of the photometry for this system. In addition to the 61 epochs of SDSS Stripe 82 photometry, we obtained follow-up $i$-band photometry from the Agile high speed imaging camera on the Astrophysical Research Consortium $3.5 \mathrm{~m}$ telescope at Apache Point Observatory (APO), and griz-band photometry from the New Mexico State University (NMSU) $1 \mathrm{~m}$ telescope.

The SDSS photometry comes from the "Stripe 82 " region, a narrow strip located along the celestial equator $\left(20^{\mathrm{h}} 24^{\mathrm{m}}<\right.$ $\left.\alpha_{\mathrm{J} 2000}<04^{\mathrm{h}} 08^{\mathrm{m}},\left|\delta_{\mathrm{J} 2000}\right|<1.266\right)$ that has been repeatedly observed throughout the course of the SDSS. This catalog produced light curves for almost eight million point sources with between $\sim 20$ and $\sim 80$ epochs. These data have been used for a variety of investigations, including studies of supernovae (Frieman et al. 2008), stellar flares (Kowalski et al. 2009), and characterizing multiwavelength point source variability (Sesar et al. 2007). SDSS J001641-000925 was observed 61 times in all five photometric bands.

The Agile camera observed the system for $\sim 45 \%$ of the orbital period $(2.2 \mathrm{hr})$ on 2008 October 30, with a cadence of $90 \mathrm{~s}$, and a median photometric error of $0.004 \mathrm{mag}$. The $\sim 0.2$ mag sinusoidal variation in the Agile light curve appeared in phase with the sparsely sampled Stripe 82 data (see Figure 1).

Multi-band follow-up was provided by the automated NMSU $1 \mathrm{~m}$ telescope (Holtzman et al. 2010). These griz data were taken over a series of 16 half-nights in 2010 from November 13 to December 2. The observing strategy and exposure times varied between the 16 nights. During two nights we imaged the binary with consecutive griz filter exposures. Single filters were used for each of the remaining 14 nights, broken down as 


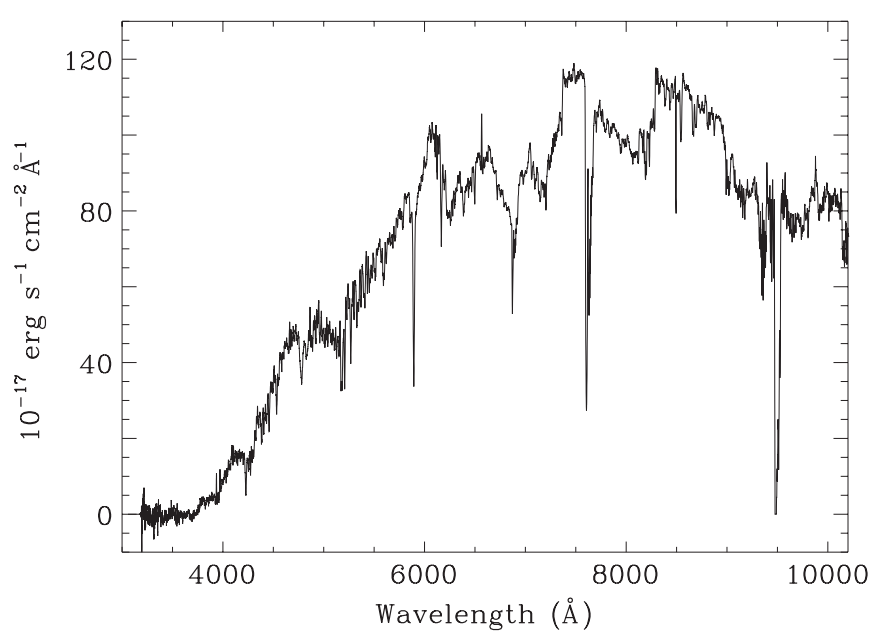

Figure 2. One of the nine MagE spectra for SDSS J001641.03-000925.2, taken at a phase of $\phi=0.236$. The spectrum has been smoothed using a boxcar kernel of 7 pixels.

follows: 2 nights of the $g$ band, 7 nights of the $r$ band, 3 nights of the $i$ band, and 2 nights of the $z$ band. These phased light curves matched the well-defined sinusoidal trends seen in the griz bands from the SDSS data, with no major deviations, e.g., from flares or starspots.

\subsection{Spectroscopy}

SDSS spectra are the result of co-adding three to six individual frames, each with 9-15 minute exposure times (York et al. 2000). The time-domain SDSS spectra have previously been examined to search for emission line variability in M dwarfs (e.g., Hilton et al. 2010; Kruse et al. 2010; Bell et al. 2012), and quasar variability (Vanden Berk et al. 2004). We obtained three time resolved spectra from 2002 October 12 using the SDSS Data Archive Server. ${ }^{6}$ No spectral line splitting was apparent from the $R \sim 1800$ resolution SDSS spectrum for this system. A single $\mathrm{H} \alpha$ emission line was seen in each frame, but no radial velocity shift was resolved for any spectral feature.

Initial follow-up spectroscopy was provided by the $6.5 \mathrm{~m}$ Magellan Clay telescope at Las Campanas Observatory, using the $R \sim 4100$ resolution Magellan Echellette Spectrograph (MagE) on 2008 October 25. We obtained nine spectra with exposure times ranging from 600 to $900 \mathrm{~s}$. These data were reduced using the MagE Spectral Extractor software package for IDL (MASE; Bochanski et al. 2009), and an example is shown in Figure 2. The observations spanned $\sim 44 \%$ of the orbital phase.

Additional spectroscopy was obtained with the APO $3.5 \mathrm{~m}$ Dual Imaging Spectrograph (DIS), using the R1200 grating with a resolution of $R \sim 3500$, on 2011 October 20. These eight spectra had integration times of $900 \mathrm{~s}$, and were timed to cover $\sim 50 \%$ of the orbital phase that was not observed with MagE.

\section{MODELING THE SYSTEM}

Using the M dwarf spectral templates from Bochanski et al. (2007b), we estimated the spectral type for both stars in this system. We scaled these template spectra, from types M0 to M3, by their approximate bolometric luminosities (Reid \& Hawley 2005), and created a normalized binary template spectrum for

\footnotetext{
6 http://das.sdss.org
}

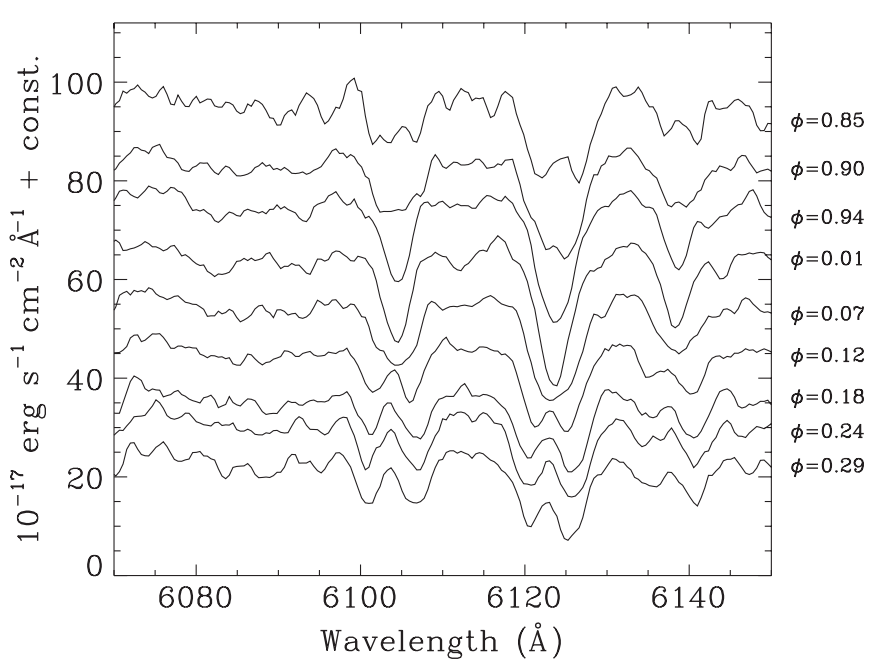

Figure 3. Nine MagE spectra showing clear splitting in the Ca I lines at 6102 and $6122 \AA$. The MagE spectra were taken in order from top to bottom, and cover $44 \%$ of the orbital phase, from $\phi=-0.15$ to 0.29 .

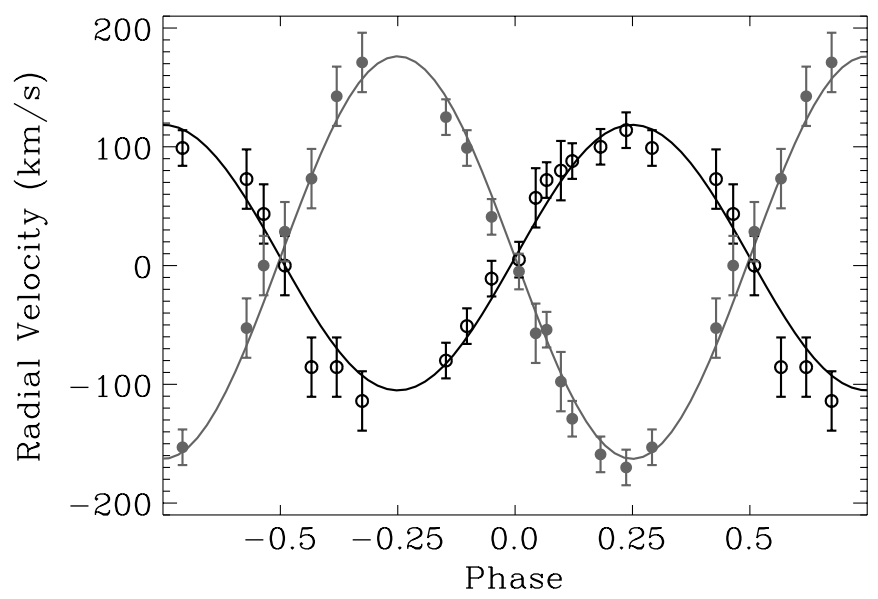

Figure 4. Radial velocities as a function of orbital phase for both components of this system for the MagE and DIS data. The velocities for the primary star are open black circles and that for the secondary star are solid gray circles. The best-fit model, as generated by the PHOEBE software, is overlaid.

each of the 16 possible combinations of stars (M0+M0, M0+M1, ..., M3+M3). The average best-fit template combination for the DIS and MagE data was M0+M1, which we adopted to seed the initial binary model.

Velocities were measured for both stellar components by cross-correlating the active M0 and M1 spectral templates from Bochanski et al. (2007b) to each of the MagE and DIS spectra using a two-dimensional cross-correlation code employing the TODCOR algorithm (Zucker \& Mazeh 1994). Figure 3 presents three absorption lines in the MagE data that show clear radial velocity splitting, indicative of a binary system. Velocities were also extracted from the spectra using the IDL broadening function code from Rucinski (2002), and were consistent with the cross-correlation results within the $\sim 15 \mathrm{~km} \mathrm{~s}^{-1}$ TODCOR uncertainties. The radial velocity curve for the MagE and DIS data is shown in Figure 4 as a function of orbital phase, with the best-fit binary model (described below) overlaid. 

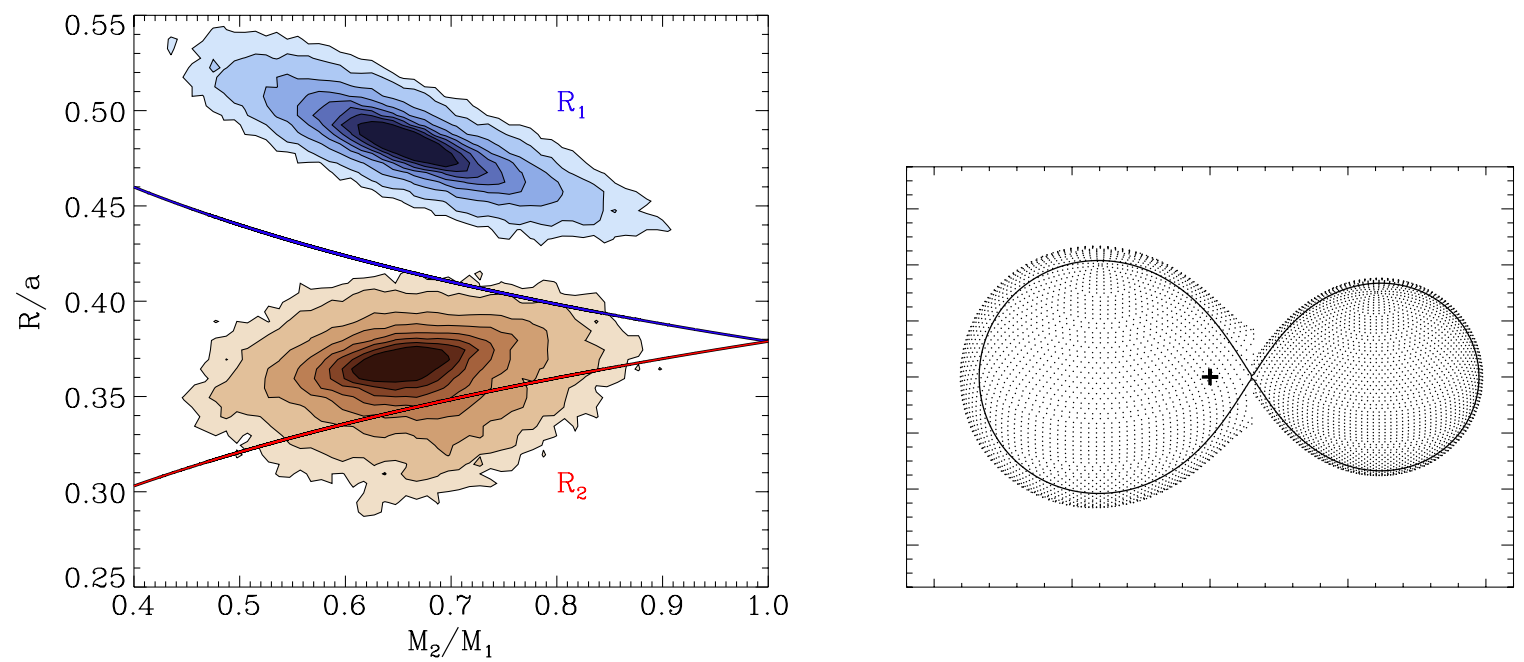

Figure 5. Top: probability distributions of the radii normalized by the semi-major axis, as a function of the mass ratio, for the primary star (blue contours) and secondary star (red contours) explored by the MCMC sampler using the Unconstrained mode in PHOEBE. The solid blue and red lines show the effective Roche lobe radii from Eggleton (1983) for the primary and secondary stars, respectively. The effective radii for both stars are larger than the critical Roche lobe radii, indicating an over-contact binary scenario. Bottom: a three-dimensional model for SDSS J001641-000925 at a phase of $\phi=0.25$ from the PHOEBE software using our best-fit values, with the primary star (left), secondary star (right), center of mass (cross), and the critical Roche equipotential (solid line).

(A color version of this figure is available in the online journal.)

\subsection{Initial Binary Model}

In order to combine the many sources of data, we used the latest stable build of the $\mathrm{PHOEBE}^{7}$ modeling software (version 0.32; Prša \& Zwitter 2005). This implementation of the Wilson \& Devinney (1971) algorithm is an industry standard in modeling binaries, and is actively being developed and improved for use in modern binary studies (e.g., Prša et al. 2011) from missions such as the Kepler space telescope (Borucki et al. 2010).

Due to the very short orbital period of the system we initially used the "Undetermined" binary configuration in the PHOEBE software, which allowed solutions wherein both stars could potentially overflow their Roche lobes. We estimated the initial system parameters assuming a M0+M1 binary pair, as found by our spectroscopic fit described above, with masses of 0.6 and $0.5 M_{\odot}$, temperatures of 3800 and $3600 \mathrm{~K}$, and an orbital separation of $1.4 R_{\odot}$.

The binary parameters were converged manually using the "Differential Corrections" solution mode. We started by fitting only the photometry, assuming $70^{\circ}$ inclination and a circular orbit. The light curves for the griz bands were fit simultaneously, and the effective temperatures and luminosities of the stars were estimated. The radial velocities from the MagE and DIS data were then included in the model, and the solution recomputed. The orbital parameters were then allowed to vary, and the solution was recomputed again using both the velocities and photometry as constraints. Eccentricity remained fixed at 0 . The orbital period from B11 for the system was adopted, with no time derivative or phase shift in the period. A logarithmic limbdarkening profile, included with PHOEBE, was used. No surface spots were included in our binary solution.

\section{2. $M C M C$}

The result of our initial binary solution indicated that the system was in a contact configuration, with the primary star overflowing its Roche lobe. This would make SDSS

\footnotetext{
7 http://phoebe.fiz.uni-lj.si/
}

J001641-000925 the first contact M dwarf binary system yet characterized. To determine the reliability of the contact solution, we employed a Markov Chain Monte Carlo (MCMC) parameter space search method.

We used the Python-based MCMC sampler emcee (ForemanMackey et al. 2012) to adjust the free parameters $T_{1}, T_{2}, a, i$, $v_{\text {sys }}$, and the potentials for the two stars $\Omega_{1}$ and $\Omega_{2}$ (Kopal 1959). Initial uncertainties for our MCMC runs were determined using the error prescription included in the PHOEBE version 0.32 user manual for our initial binary model parameters. We then used the command line PHOEBE "Scripter" to compute the $\chi^{2}$, masses, and radii for each iteration (step).

A total of 16 parameter space "Walkers" were used, and multi-threading with 7 computer cores was implemented using the Python multiprocessing module. The first 1000 steps were discarded, to allow the sampler an initial exploration of the parameter space. The MCMC was run for an additional 20,000 steps for each Walker, for a total of 320,000 iterations.

We ran our MCMC search using both the "Overcontact Binary of the W UMa type" mode and "Unconstrained" mode in PHOEBE, as the constraints for each mode can affect the resulting parameter estimates, especially of the radii (e.g., Bonanos 2009; Caballero-García et al. 2010). The best-fit results for the free parameters in both modes were the same within the uncertainties. The secondary star radius, however, was very different between the modes. In Figure 5 we show the radii versus mass ratio probability distributions, and a threedimensional illustration of the system at a phase of $\phi=0.25$, with the Unconstrained mode MCMC runs. Figure 6 shows the MCMC results using the W UMa Overcontact mode. We note that the Overcontact model (Wilson \& Devinney 1971; Wilson 2001) can converge on a physically incorrect solution (e.g., see Pribulla \& Rucinski 2008), particularly for systems with mass transfer or mass loss as discussed in Section 4.2. Both modes, however, indicated that the system is in contact (Rucinski 1997; Wilson 2001), and we present results for the Overcontact W UMa mode as our preferred final model. The stars were found to have masses of $M_{1}=$ $0.54 \pm 0.07 M_{\odot}$ and $M_{2}=0.34 \pm 0.04 M_{\odot}$, which correspond 

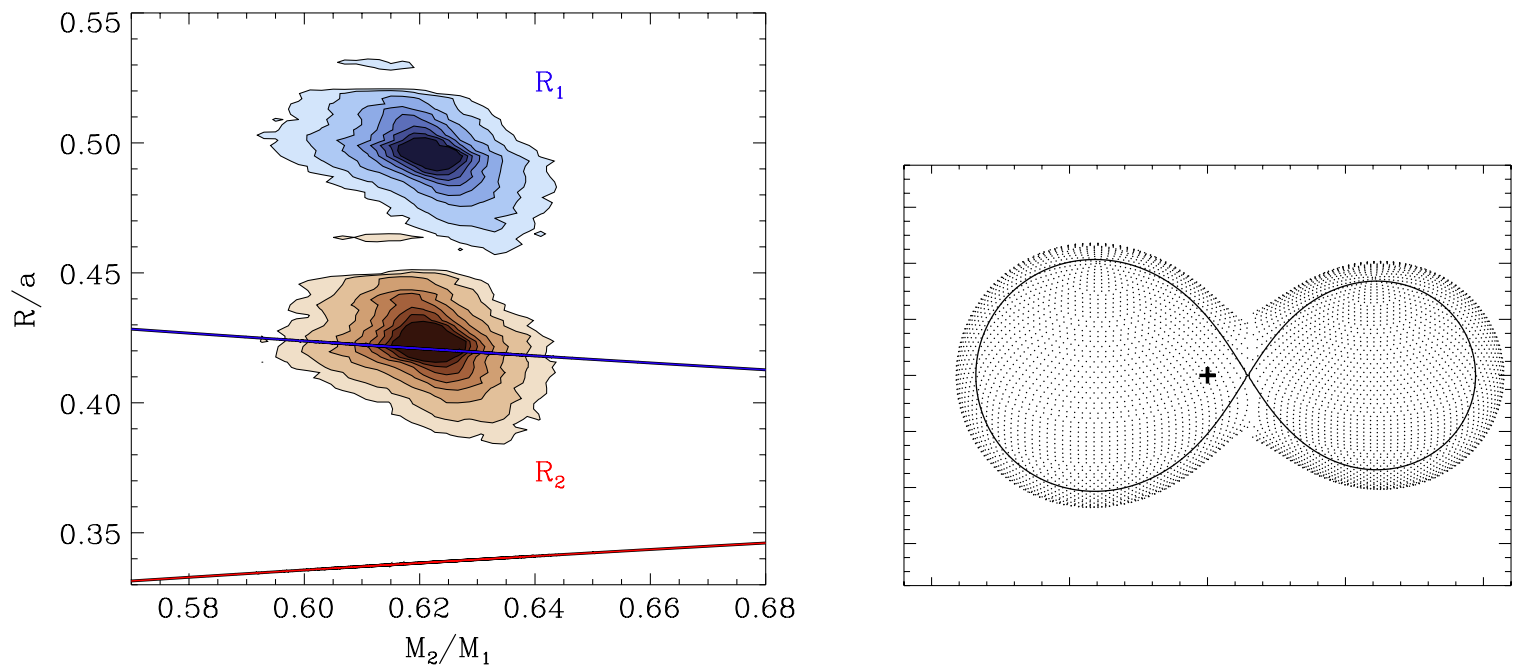

Figure 6. Same as Figure 5, but for the W UMa Overcontact mode in PHOEBE.

(A color version of this figure is available in the online journal.)

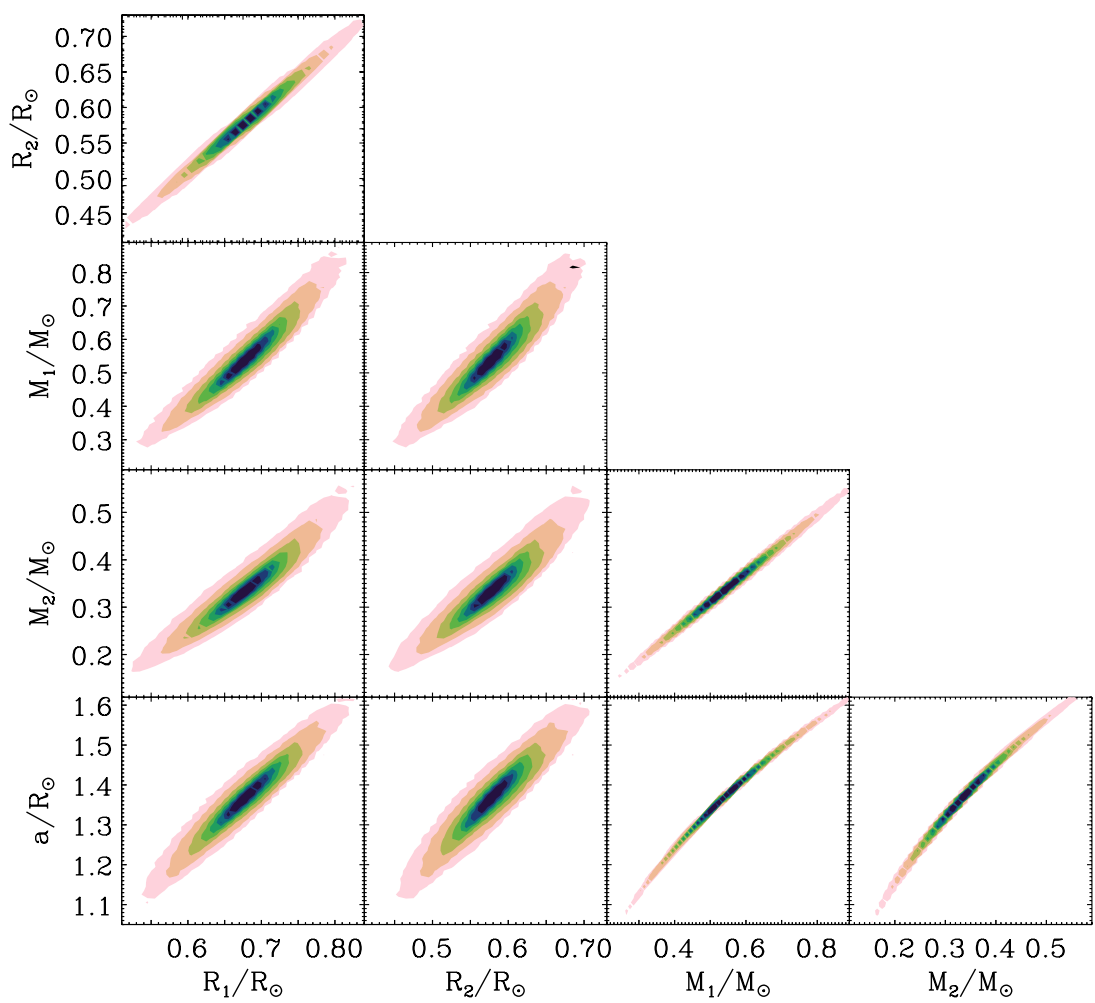

Figure 7. Probability distributions for the masses, radii, and the semi-major axis for SDSS J001641-000925 as explored by our MCMC sampler using the W UMa Overcontact mode in PHOEBE.

(A color version of this figure is available in the online journal.)

to spectral types M1 and M3 (Reid \& Hawley 2005), and radii of $R_{1}=0.68 \pm 0.03 R_{\odot}$ and $R_{2}=0.58 \pm 0.03 R_{\odot}$. A grid of the resulting probability distributions for the masses, radii, and semi-major axis is shown in Figure 7.

We computed the correlation lengths for each of our free parameters using the autocorrelation function. The effective chain length is defined as the actual length of the MCMC chain after the 1000 step "burn-in," divided by the correlation length, and must be $\gg 1$ for meaningful results (Tegmark et al. 2004). The shortest effective chain length in our study was for the inclination parameter, with a value of 7106 . All other free parameters had effective chain lengths over 9000 , indicating that our parameter space sampling reliably converged on the correct solution, and that the resulting errors were realistic. We also tested the convergence of our MCMC runs with the Gelman-Rubin $\hat{R}$ statistic, as detailed in Section 3.3 of Ford (2006). All parameters converged to $\hat{R}<$ 1.001 , indicating our MCMC effectively sampled the parameter space.

The final radial velocity solution is shown in Figure 4, and the SDSS phase curves computed from the final PHOEBE model are shown in Figure 8. The median model values for the system 


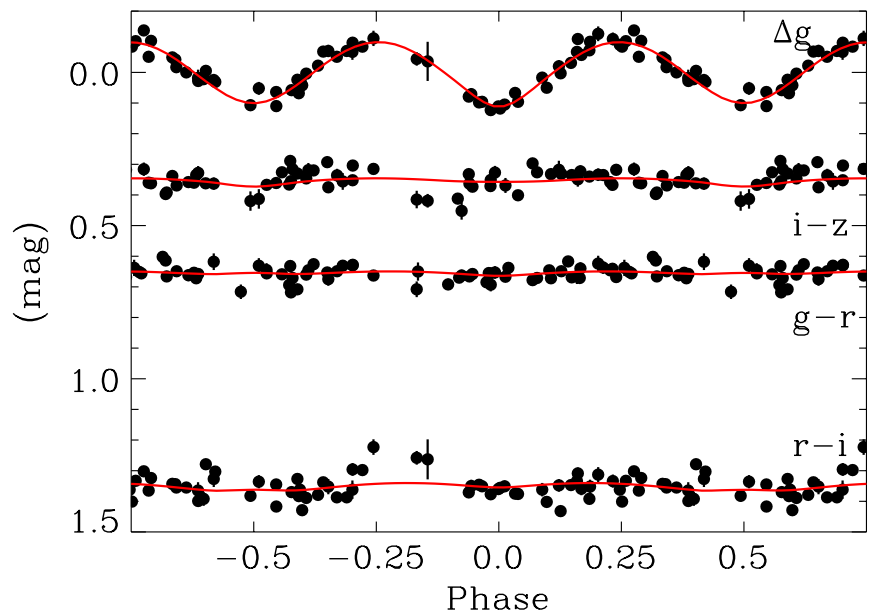

Figure 8. Differential SDSS $g$-band, $(g-r)$, and $(r-i)$ colors as a function of orbital phase, along with the PHOEBE model fit (red).

(A color version of this figure is available in the online journal.)

Table 1

The System Parameters and Uncertainties for SDSS J001641-000925 Determined by Our MCMC Runs

\begin{tabular}{lcc}
\hline \hline Quantity & Value & Uncertainty \\
\hline$P$ (days) & 0.198561 & $1 \times 10^{-6}$ \\
$t_{0}($ days $)$ & 0.002405 & $1 \times 10^{-6}$ \\
$M_{1}\left(M_{\odot}\right)$ & 0.54 & 0.07 \\
$M_{2}\left(M_{\odot}\right)$ & 0.34 & 0.04 \\
$R_{1}\left(R_{\odot}\right)$ & 0.68 & 0.03 \\
$R_{2}\left(R_{\odot}\right)$ & 0.58 & 0.03 \\
$T_{\text {eff, }}(\mathrm{K})$ & 4342.0 & 475.0 \\
$T_{\text {eff, }}(\mathrm{K})$ & 3889.0 & 579.0 \\
$a\left(R_{\odot}\right)$ & 1.37 & 0.06 \\
$q\left(M_{2} / M_{1}\right)$ & 0.62 & 0.01 \\
$i\left(^{\circ}\right)$ & 53.3 & 1.1 \\
$v_{\text {sys }}\left(\mathrm{km} \mathrm{s}^{-1}\right)$ & -5.6 & 3.9 \\
\hline
\end{tabular}

parameters are given in Table 1. Errors for each parameter were determined using the standard deviation of the probability distributions.

\section{RESULTS}

\subsection{Binary System Parameters}

The illustration of our binary system in Figure 6 clearly indicates that both stars are filling their Roche lobes. We also show the probability distributions for the normalized radii of both stars. Overlaid is the critical Roche radius for both the primary and secondary stars, calculated using the Eggleton (1983) approximation. The MCMC probability distribution for the W UMa model mode showed both stars to be larger than their critical Roche radius with $100 \%$ confidence. The primary star was $18 \%$ larger than the critical Roche radius, and the secondary star $25 \%$ larger. This raises the intriguing possibility that the system is in over-contact (Rucinski 1997), in which case we would expect to see non-solid body velocities from outflowing material leaving the system via the L2 point. This would be a very efficient method of angular momentum loss for the binary, and could lead to rapid decay of its orbit.

The masses and radii of SDSS J001641-000925 with respect to other low-mass eclipsing binaries are plotted in Figure 9. Bayless \& Orosz (2006) provided an empirical fit to the mass-radius relation using many low-mass binary systems with

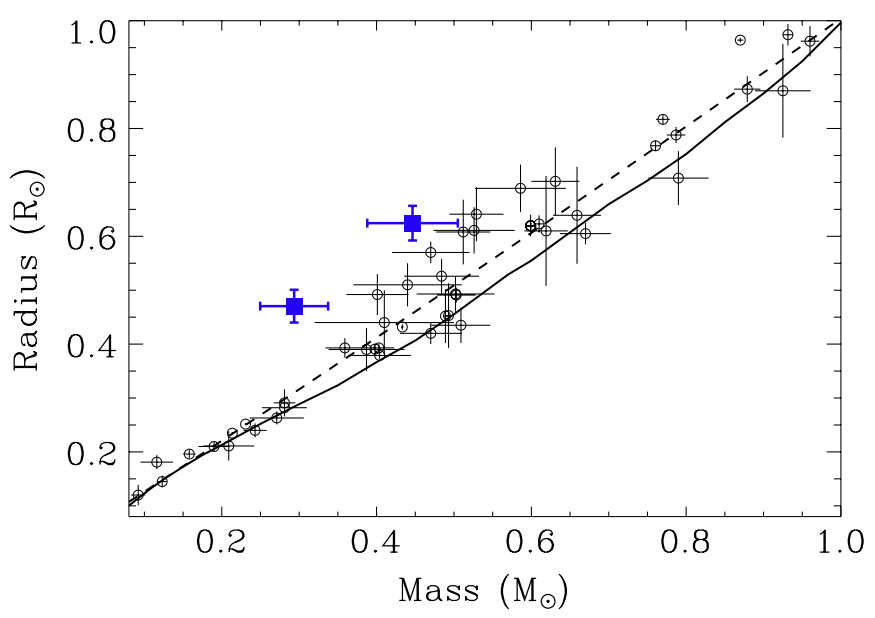

Figure 9. Stellar radius as a function of mass for the compilation of single and binary stars from López-Morales (2007), as well as the system from Becker et al. (2008), in open black circles. Our PHOEBE solution for SDSS J001641-000925 is shown as filled blue squares. The solid black line is the Baraffe et al. (1998) stellar evolution model, and the dashed line is the empirical fit from Bayless \& Orosz (2006).

(A color version of this figure is available in the online journal.)

larger than predicted radii. Our system is much larger than the Bayless \& Orosz (2006) prediction at this mass range, due to the tidal distortion effects of the over-contact solution.

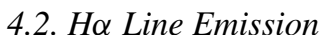

$\mathrm{H} \alpha$ emission from the system was present in every observed spectrum. This is not an uncommon property of $\mathbf{M}$ dwarfs in general, whose magnetic activity is frequently characterized by strong and variable $\mathrm{H} \alpha$ line emission (e.g., West et al. 2011; Bell et al. 2012). However, the fraction of magnetically active field stars with spectral types M2 or earlier is less than $10 \%$ (West et al. 2011). The strength and frequency of this emission decrease as $M$ dwarfs age, and the lifetime of magnetic activity for early-type $\mathrm{M}$ dwarfs in the field is less than $\sim 2$ Gyr (West et al. 2008). $\mathrm{H} \alpha$ emission is also seen from $\mathbf{M}$ dwarfs in close binary systems with other dwarf stars (e.g., Dimitrov \& Kjurkchieva 2010) and with white dwarfs (Maxted et al. 1998; Silvestri et al. 2006). The higher fraction of active early-type M dwarfs in close binaries as compared to the field is likely due to these stars rotating more rapidly than their isolated counterparts, inducing stronger and longer lived magnetic activity (Morgan et al. 2012; Silvestri et al. 2006).

We measured the $\mathrm{H} \alpha$ equivalent width (EW) for each DIS, MagE, and SDSS spectra, shown in Figure 10(a) as a function of orbital phase. The strength of emission is nearly constant at $\sim 1 \AA$, though a weak correlation in the line strength was found with orbital phase, with a higher $\mathrm{H} \alpha \mathrm{EW}$ at conjuncture $(\phi=0$ and 0.5).

The $\mathrm{H} \alpha$ line was observed to be broader than other features (e.g., the Ca I absorption lines in Figure 3), and clear splitting was only seen in a few spectra. The feature did not contribute significantly to the cross-correlation in Section 3, which was dominated by absorption features such as those shown in Figure 3. We measured the radial velocity of the $\mathrm{H} \alpha$ line, shown in Figure 10(b), by fitting two Gaussian profiles to the emission feature. The $\mathrm{H} \alpha$ line broadly followed the velocity trend of the more massive stellar component, possibly due to the primary dominating the total $\mathrm{H} \alpha$ flux. Line splitting was seen near quadrature $(\phi=0.25)$ in the higher resolution MagE spectra, 


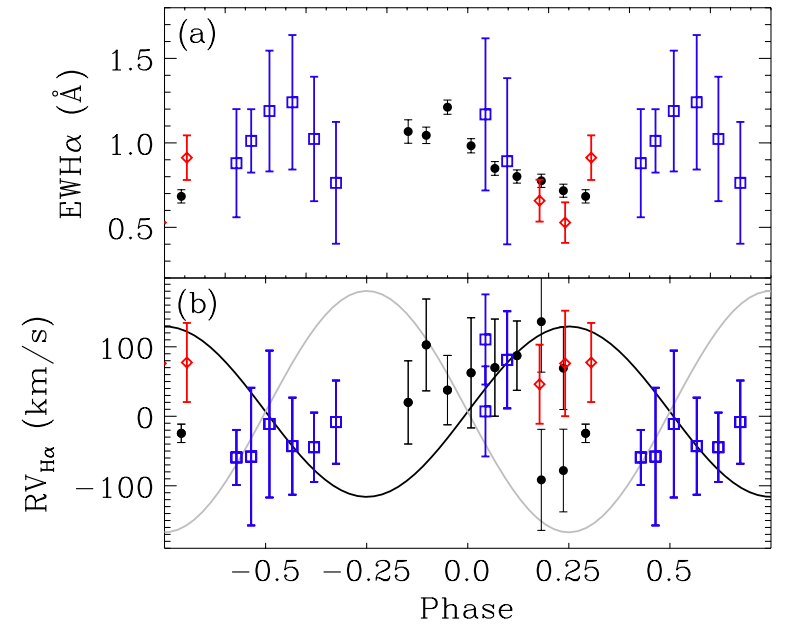

Figure 10. (a) The equivalent width of the $\mathrm{H} \alpha$ line in each spectrum as a function of orbital phase for the MagE (filled black circles), DIS (open blue squares), and SDSS time spectra (open red diamonds). (b) The radial velocity of the $\mathrm{H} \alpha$ line for each spectrum, with the orbital radial velocity solution from Figure 4 overlaid for comparison, using the same color coding and symbols as above.

(A color version of this figure is available in the online journal.)

and the $\mathrm{H} \alpha$ velocity profiles are consistent with emission from both stellar components in this system.

No other evidence for accretion, flaring, or outflow in the form of nebular emission line features was observed. The line splitting seen in the MagE data at $\phi=0.25$ indicates that both stars are magnetically active. We would conventionally assume the $\mathrm{H} \alpha$ emission comes from many distributed active regions on the stellar surfaces. However, the broadened $\mathrm{H} \alpha$ emission, lower amplitude radial velocities, and intermittent line splitting suggests non-solid body velocities, and may indicate mass transfer between the stars and mass loss, likely through the L2 point as a result of the over-contact configuration. The slight increases in $\mathrm{H} \alpha \mathrm{EW}$ at conjuncture are reminiscent of those from the massive over-contact binary system RY Scuti, which are attributed to extended $\mathrm{H} \alpha$ emission from outflows (Grundstrom et al. 2007) that appear to have brighter EW during eclipse $(\phi=0$ and $\phi=0.5)$ when the system flux is lower. Higher resolution spectroscopic monitoring throughout the orbital period is necessary to accurately determine the geometry of the $\mathrm{H} \alpha$ emission regions, and the possible active region timescales.

\subsection{Period Decay}

The observed period of a close binary system should decrease over time, as the orbit decays and the system loses angular momentum from effects such as magnetic braking, tidal friction, mass transfer, mass loss, and in the most compact cases gravitational waves. Changes in the orbital period have been observed for several hundred W-UMa-type binary systems. Pilecki et al. (2007) measured period decreases for $\sim 30$ short period systems, finding typical amplitudes of $\dot{P} \equiv d P / d t \sim$ $-1 \mathrm{~s} \mathrm{yr}^{-1}$. Kubiak et al. (2006) searched the OGLE database (Udalski et al. 1992) for period changes from more than 500 short period binaries, finding a maximum amplitude of $\dot{P}=-0.4 \mathrm{~s} \mathrm{yr}^{-1}$.

The SDSS Stripe 82 data were very sparsely sampled in time, and individual times of light curve minima were not observed. As such, we were unable to make a traditional observed minus computed $(O-C)$ diagram. Instead we broke the SDSS data

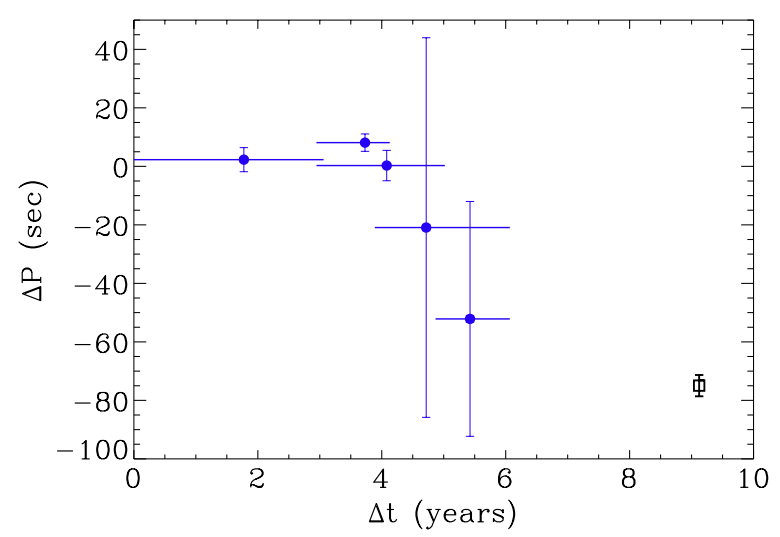

Figure 11. Difference in orbital period between the B11 value and our measurements, using SDSS (blue) and NMSU (black) photometry, as a function of time. Periods shorter than the B11 value yield negative $\Delta P$. The first SDSS epoch, at $\Delta t=0$, had an MJD $=51075.316$. Horizontal bars give the range of data for each SDSS subset, with each point placed at the mean epoch for the subset. Error bars were determined using a bootstrap method, described in the text.

(A color version of this figure is available in the online journal.)

into five subsets, spanning different ranges in time, each having at least 20 epochs. The NMSU $1 \mathrm{~m}$ photometry were obtained in a single observing season, with many full orbits observed, and were used as a sixth subset.

To search for changes in the orbital period of SDSS J001641-000925, we measured the period for each time interval in our data with a phase dispersion minimization code, using the SuperSmoother algorithm (Friedman 1984) as described in Reimann (1994). We folded each subset at periods ranging \pm 5 minutes around the B11 value, in steps of $0.1 \mathrm{~s}$.

In Figure 11 we show the difference in period from the B11 value for each subset of $r$-band Stripe 82 and NMSU data. The time span for each Stripe 82 subset is shown as horizontal bars. A consistently decreasing period was found, with a total amplitude of $\sim 75 \mathrm{~s}$, spanning the nine years of observations, yielding $\dot{P} \sim-8 \mathrm{~s} \mathrm{yr}^{-1}$. A similar approach was used to measure period decay for V1309 Sco in Tylenda et al. (2011).

We recomputed the period determination for each data subset using a bootstrap method, leaving one epoch out each time. Errors shown in Figure 11 are the standard deviation of the bootstrap runs. The Stripe 82 period results are somewhat dependent on the time range chosen, as shown in Figure 11. The independent period measurement from the NMSU photometry, however, provides strong evidence of orbital decay for this system, though we note the caveat that this is dominated by the NMSU photometry.

\section{DISCUSSION}

We have presented follow-up spectroscopic and photometric observations of a low-mass binary, SDSS J001641-000925, whose orbital period is below the theoretical short period limit (Rucinski 1992). The components of this short period system have masses typical of spectral types of M1 and M3, and are in an over-contact configuration. Both stars have radii much larger than predicted by stellar isochrone models. These stars are the most "oversized" of any yet determined at this mass due to the over-contact configuration.

The strength of the $\mathrm{H} \alpha$ line at $\sim 1 \AA$ was not particularly high with respect to active stars of this spectral type. Higher $\mathrm{H} \alpha$ flux is typically accompanied by strong magnetic activity and 
flaring for early-type $\mathrm{M}$ dwarfs; these are features that diminish as the stars age and lose angular momentum (West et al. 2008). The SDSS spectroscopic M dwarf sample, containing 70,000 spectroscopically confirmed M dwarfs (West et al. 2011) shows approximately half of stars with spectral types M0-M3 that are classified as "active" have $\mathrm{EW}(\mathrm{H} \alpha) \geqslant 1.25 \AA$. Thus the $\mathrm{H} \alpha \mathrm{EW}$ for this system is not usually high, nor does it appear particularly active. In fact, no signs of flaring were detected in more than 20 nights of observing. This is in strong contrast to the similar mass semi-detached binary system observed by Dimitrov \& Kjurkchieva (2010), which showed six flares in a comparable amount of observing time. Davenport et al. (2012) found that field stars in this spectral type range displayed photometric flares with amplitudes of $\Delta u \geqslant 1$ mag once every $\sim 2$ days on average.

However, the slight increase in $\mathrm{H} \alpha \mathrm{EW}$ during eclipse, as well as the broadened $\mathrm{H} \alpha$ line profile with possibly non-solid body rotation velocities, together suggest that the emission feature may be the result of material being expelled from the outer Lagrange points. This would result in a complex $\mathrm{H} \alpha$ line, with phase-dependent emission lines contributed from both stars, as well as possible P Cygni-like profiles from the outflowing material. Additional medium- to high-resolution spectroscopic monitoring will allow us to differentiate $\mathrm{H} \alpha$ emission due to active surface regions from that of any nebular emission surrounding the system, as in RY Scuti (Grundstrom et al. 2007).

Dynamical interactions between a binary system and other stars can decrease the binary orbital period through angular momentum transfer, or through three-body interactions (Kozai 1962). Indeed the incidence of contact binary systems is higher in stellar clusters than in the field (Rucinski 1998), due to the more frequent three-body interactions. Recent models predict a timescale of 6-13 Gyr for an isolated low-mass binary system to undergo enough angular momentum loss to evolve from a stable orbital period of $\sim 1-2$ days to below 0.22 days (Stepien 2006a, 2006b). Estimates of angular momentum loss from single stars, applied to short period binary systems, show that magnetic braking takes on the order of a Hubble time to compress these periods from one to two days to below the theoretical limit (e.g., Maceroni \& Montalbán 2004). With their discovery of several low-mass systems below the predicted 0.22 day limit, Nefs et al. (2012) demonstrated that initial orbital periods must have been $\sim 1$ day in order for magnetic braking schemes to explain the short period systems. Nefs et al. (2012) also show that the orbital evolutionary timescales and initial conditions for these rare short period binaries are still largely unconstrained, and that many possible mechanisms may be at work in forming such systems. Once the stars reach a contact scenario, unstable mass transfer via the filled Roche lobes, and mass loss through the outer Lagrange points, predicts a rapid evolution toward coalescence (Jiang et al. 2011). This type of over-contact evolution (Rucinski 1997 ) is thought to be a formation path for blue straggler stars in stellar clusters (Bradstreet \& Guinan 1994; Mateo et al. 1990), and signatures include mass loss, changes in luminosity, and orbital decay.

Our measured period decay of approximately $|\dot{P}| \sim 8 \mathrm{~s}$ each year is larger than found for typical W UMa binary systems. The largest amplitude period decay measured for a contact binary is that of V1309 Sco, a merger system with an exponentially decreasing period with an amplitude of $|\dot{P}| \sim 300 \mathrm{~s} \mathrm{yr}^{-1}$ (Tylenda et al. 2011). This system exhibited an extreme nova-like outburst, similar to V838 Mon, as it underwent a catastrophic merging event in 2008. Assuming a linear period decay of $|\dot{P}| \sim 8 \mathrm{~s} \mathrm{yr}^{-1}$, we would expect SDSS J001641-000925 to have a lifetime of $\sim 10^{3}$ years. However, if the period evolution follows an exponential decay as in the higher mass V1309 Sco system, the binary merger timescale may be as short as $\sim 10^{2}$ years.

This amplitude of angular momentum loss required by the observed period decay cannot be explained solely by magnetic braking, and the stars are too widely separated for gravitational waves to expel significant energy (Chau 1978). The angular momentum loss is likely due to the system being in an overcontact configuration and having filled its critical Roche surface, with mass being expelled from the outer Lagrangian points. This would carry much more angular momentum from the system than magnetic breaking or winds alone, and would lead to a rapid coalescence into a single star ( $\mathrm{Li}$ et al. 2004). However, we cannot exclude the possibility of mass transfer from the primary to the secondary star with little to no loss of angular momentum to the system. This mass redistribution could instead lead to a binary evolution as described by Stepien (2006a), with the orbital period increasing, and binary coalescence proceeding on a much more gradual nuclear or thermal timescale ( $\mathrm{Li}$ et al. 2004). Our follow-up study of the period evolution will help discern between these two possibilities.

We emphasize the rarity of this system, as SDSS J001641-000925 is the first true over-contact M dwarf binary known. The critical question remaining for the system is whether it is evolving rapidly toward coalescence due to the over-contact configuration. Additional high precision follow-up photometry, as well as searching existing time domain surveys, is underway to conclusively determine whether the period is decaying at an exponential rate, as was the case for V1309 Sco before its dramatic merger.

The authors thank Dr. John Wisniewski for fruitful discussions of low-mass stars in binary systems, Dr. Adam Kowalski and Dr. Sarah Schmidt for assistance with observing, Mr. John Ruan for help with Python, and the referee, Dr. Slavek Rucinski, for comments and suggestions that greatly improved this manuscript. J.R.A.D. and A.C.B. acknowledge support from NASA ADP grant NNX09AC77G. S.L.H. and E.J.H. acknowledge support from NSF grant AST 0807205.

This publication makes use of data products from the Two Micron All Sky Survey, which is a joint project of the University of Massachusetts and the Infrared Processing and Analysis Center/California Institute of Technology, funded by the National Aeronautics and Space Administration and the National Science Foundation.

Funding for SDSS-III has been provided by the Alfred P. Sloan Foundation, the Participating Institutions, the National Science Foundation, and the U.S. Department of Energy Office of Science. The SDSS-III Web site is http://www.sdss3.org/. SDSS-III is managed by the Astrophysical Research Consortium for the Participating Institutions of the SDSS-III Collaboration including the University of Arizona, the Brazilian Participation Group, Brookhaven National Laboratory, University of Cambridge, University of Florida, the French Participation Group, the German Participation Group, the Instituto de Astrofisica de Canarias, the Michigan State/Notre Dame/ JINA Participation Group, Johns Hopkins University, Lawrence Berkeley National Laboratory, Max Planck Institute for Astrophysics, New Mexico State University, New York University, Ohio State University, Pennsylvania State University, University of Portsmouth, Princeton University, the Spanish Participation 
Group, University of Tokyo, University of Utah, Vanderbilt University, University of Virginia, University of Washington, and Yale University.

\section{REFERENCES}

Baraffe, I., Chabrier, G., Allard, F., \& Hauschildt, P. H. 1998, A\&A, 337, 403 Bayless, A. J., \& Orosz, J. A. 2006, ApJ, 651, 1155

Becker, A. C., Agol, E., Silvestri, N. M., et al. 2008, MNRAS, 386, 416

Becker, A. C., Bochanski, J. J., Hawley, S. L., et al. 2011, ApJ, 731, 17 (B11)

Bell, K. J., Hilton, E. J., Davenport, J. R. A., et al. 2012, PASP, 124, 14

Bochanski, J. J., Hawley, S. L., Covey, K. R., et al. 2010, AJ, 139, 2679

Bochanski, J. J., Hawley, S. L., \& West, A. A. 2011, AJ, 141, 98

Bochanski, J. J., Hennawi, J. F., Simcoe, R. A., et al. 2009, PASP, 121, 1409

Bochanski, J. J., Munn, J. A., Hawley, S. L., et al. 2007a, AJ, 134, 2418

Bochanski, J. J., West, A. A., Hawley, S. L., \& Covey, K. R. 2007b, AJ, 133,531

Bonanos, A. Z. 2009, ApJ, 691, 407

Borucki, W. J., Koch, D., Basri, G., et al. 2010, Sci, 327, 977

Bradstreet, D. H., \& Guinan, E. F. 1994, in ASP Conf. Ser. 56, Interacting Binary Stars, ed. A. W. Shafter (San Francisco, CA: ASP), 228

Caballero-García, M. D., Torres, G., Ribas, I., et al. 2010, A\&A, 514, A36

Chabrier, G., Gallardo, J., \& Baraffe, I. 2007, A\&A, 472, L17

Chau, W. Y. 1978, ApJ, 219, 1038

Covey, K. R., Ivezić, Ž., Schlegel, D., et al. 2007, AJ, 134, 2398

Davenport, J. R. A., Becker, A. C., Kowalski, A. F., et al. 2012, ApJ, 748, 58

Dimitrov, D. P., \& Kjurkchieva, D. P. 2010, MNRAS, 406, 2559

Eggleton, P. P. 1983, ApJ, 268, 368

Ford, E. B. 2006, ApJ, 642, 505

Foreman-Mackey, D., Hogg, D. W., Lang, D., \& Goodman, J. 2012, arXiv: 1202.3665

Friedman, J. H. 1984, A Variable Span Smoother, Tech. Rep. 5, Department of Statistics, Stanford Univ.

Frieman, J. A., Bassett, B., Becker, A., et al. 2008, AJ, 135, 338

Grundstrom, E. D., Gies, D. R., Hillwig, T. C., et al. 2007, ApJ, 667, 505

Hilton, E. J., West, A. A., Hawley, S. L., \& Kowalski, A. F. 2010, AJ, 140, 1402

Holtzman, J. A., Harrison, T. E., \& Coughlin, J. L. 2010, AdAst, 2010, 193086

Ivezić, Ž., Smith, J. A., Miknaitis, G., et al. 2007, AJ, 134, 973

Jiang, D., Han, Z., Ge, H., Yang, L., \& Li, L. 2011, ArXiv e-prints

Jiang, D., Han, Z., Ge, H., Yang, L., \& Li, L. 2012, MNRAS, 421, 2769

Kopal, Z. 1959, Close Binary Systems (London: Chapman \& Hall)

Kowalski, A. F., Hawley, S. L., Hilton, E. J., et al. 2009, AJ, 138, 633
Kozai, Y. 1962, AJ, 67, 591

Kruse, E. A., Berger, E., Knapp, G. R., et al. 2010, ApJ, 722, 1352

Kubiak, M., Udalski, A., \& Szymanski, M. K. 2006, AcA, 56, 253

Lada, C. J. 2006, ApJL, 640, L63

Li, L., Han, Z., \& Zhang, F. 2004, MNRAS, 355, 1383

López-Morales, M. 2007, ApJ, 660, 732

Maceroni, C., \& Montalbán, J. 2004, A\&A, 426, 577

Maceroni, C., \& Rucinski, S. M. 1997, PASP, 109, 782

Mateo, M., Harris, H. C., Nemec, J., \& Olszewski, E. W. 1990, AJ, 100, 469

Maxted, P. F. L., Marsh, T. R., Moran, C., Dhillon, V. S., \& Hilditch, R. W. 1998, MNRAS, 300, 1225

Mazeh, T. 2008, in EAS Publ. Ser. 29, Tidal Effects in Stars, Planets, and Disks, ed. M.-J. Goupil \& J.-P. Zahn (Cambridge: Cambridge Univ. Press), 1

Morales, J. C., Gallardo, J., Ribas, I., et al. 2010, ApJ, 718, 502

Morgan, D. P., West, A. A., Garcés, A., et al. 2012, AJ, 144, 93

Nefs, S. V., Birkby, J. L., Snellen, I. A. G., et al. 2012, MNRAS, 425, 950

Pilecki, B., Fabrycky, D., \& Poleski, R. 2007, MNRAS, 378, 757

Pribulla, T., \& Rucinski, S. M. 2008, MNRAS, 386, 377

Prša, A., Batalha, N., Slawson, R. W., et al. 2011, AJ, 141, 83

Prša, A., \& Zwitter, T. 2005, ApJ, 628, 426

Reid, I. N., \& Hawley, S. L. (ed.) 2005, New Light on Dark Stars: Red Dwarfs, Low-mass Stars, Brown Dwarfs (Springer: Praxis)

Reimann, J. D. 1994, PhD thesis, Univ. California, Berkeley

Ribas, I. 2006, Ap\&SS, 304, 89

Rucinski, S. M. 1992, AJ, 103, 960

Rucinski, S. M. 1997, IBVS, 4460, 1

Rucinski, S. M. 1998, AJ, 116, 2998

Rucinski, S. M. 2002, AJ, 124, 1746

Rucinski, S. M., \& Pribulla, T. 2008, MNRAS, 388, 1831

Sesar, B., Ivezić, Ž., Lupton, R. H., et al. 2007, AJ, 134, 2236

Silvestri, N. M., Hawley, S. L., West, A. A., et al. 2006, AJ, 131, 1674

Stepien, K. 2006a, AcA, 56, 199

Stepien, K. 2006b, AcA, 56, 347

Tegmark, M., Strauss, M. A., Blanton, M. R., et al. 2004, PhRvD, 69, 103501

Tylenda, R., Hajduk, M., Kamiński, T., et al. 2011, A\&A, 528, A114

Udalski, A., Szymanski, M., Kaluzny, J., Kubiak, M., \& Mateo, M. 1992, AcA, 42,253

Vanden Berk, D. E., Wilhite, B. C., Kron, R. G., et al. 2004, ApJ, 601, 692

West, A. A., Hawley, S. L., Bochanski, J. J., et al. 2008, AJ, 135, 785

West, A. A., Morgan, D. P., Bochanski, J. J., et al. 2011, AJ, 141, 97

Wilson, R. E. 2001, IBVS, 5076, 1

Wilson, R. E., \& Devinney, E. J. 1971, ApJ, 166, 605

York, D. G., Adelman, J., Anderson, J. E., Jr., et al. 2000, AJ, 120, 1579

Zucker, S., \& Mazeh, T. 1994, ApJ, 420, 806 